ISSN 1997-5902

\title{
An Ethnobotanical Study of Medicinal Plants of the Agadir Ida Ou Tanane Province (Southwest Morocco)
}

\author{
Ouhaddou $\mathrm{H}^{1,2}$., Boubaker $\mathrm{H}^{2}$., Msanda $\mathrm{F}^{2}$., El Mousadik A $^{2}$. \\ ${ }^{1}$ Direction Régionale des Eaux et Forêts et de la Lutte Contre la Désertification du Sud-Ouest, Agadir principale, B.P \\ 520, Agadir, Morocco. Email: halimouhaddou@gmail.com \\ ${ }^{2}$ Laboratoire de Biotechnologies et Valorisation des Ressources Naturelles, Faculté des Sciences, Cité Dakhla, B.P \\ 8106, Agadir, Morocco. Email: h.boubaker@uiz.ac.ma; f.msanda@uiz.ac.ma; a.elmousadik@uiz.ac.ma \\ Corresponding author email: halimouhaddou@gmail.com
}

Original submitted in on $6^{\text {th }}$ October 2014. Published online at www.m.elewa.org on 29th December 2014

http://dx.doi.org/10.4314/jab.v84i1.5

\begin{abstract}
Objective: As part of the development of natural heritage of Morocco, an ethnobotanical study was conducted in the region of Agadir Ida Ou Tanane (Southwest Morocco) with the aim to collect detailed information about the usage of plants in human therapy.

Methodology and results: The survey was carried out over a period of 24 months, by means of semi-structured and structured interviews. A total of 400 interviews were done with traditional health practitioners and knowledgeable villagers. Data collected was on, vernacular names of plants, their uses, parts used and mode of preparation. Other information about users was also collected such as age, sex, level of education. A total of 110 plants species belonging to 53 families and 95 genera were inventoried with $7.27 \%$ of the species endemic to Morocco. Plants frequently used were: Thymus satureioides (Tazouknnit, Zaitra), Thymus broussonnetii (Azoukni, Zaater), Argania spinosa (Argan), Tetraclinis articulata (Azouka, Aârar) and Lavandula dentate (Igerch, Halhal). The elderly (more than 50 years) have more knowledge in medicinal plants with regard to the other age groups, this indicates that knowledge was acquired by long experience accumulated.

Conclusion and application of results: This survey shows that traditional medicine is still used and constituted a very rich heritage in Agadir Ida Ou Tanane Region. The collected data may help to avoid the loss of traditional knowledge on the use of medicinal plants detained in the study area, and represent the preliminary information required in view of a future phytochemical investigation on the most used plants.
\end{abstract}

Key words: Ethnobotanical survey, medicinal plants, phytotherapy, Agadir Ida Ou Tanane province, southwest of Morocco.

\section{INTRODUCTION}

Plants are an important source of therapeutic drugs and play an important role in the survival of the tribal and ethnic communities (Abouri et al., 2012). All old civilizations (Chinese, Greek, Roman, Muslim) resorted to medicinal plants for their medicinal, aromatic properties as well as ritual uses (Younos, 1997, Ghanmi et al., 2011). Healing plants remain the only way of treatment for $70 \%$ of the world population (Rejdali, 1996, Peyron, 2000) and for 80 $\%$ of the African population (OMS, 2003). The percentage of use of healing plants in the preventive and curative virtues varies in the studied regions, from 50 to $80 \%$ (Eddouks et al., 2007). More than 25000 plants are used in the pharmacopoeia and more than $50 \%$ of pharmaceutical products available on the market are of natural origin 
(Hamilton, 2003). Medicinal plants have several advantages; they have affordable prices, are easily accessible, and there is no evidence of resistance to whole-plant extracts (Al-Adhroey et al., 2010). Morocco is one of the Mediterranean countries with a long tradition in the field of phytotherapy (Jouad et al., 2001; Scherrer et al., 2005). Many authors have studied traditional pharmacopoeia in different areas of Morocco (Bellakhdar et al., 1991; Sijelmassi, 2003; Bellakhdar, 1997; Ziyyat et al., 1997; Merzouki et al., 2000; Jouad et al., 2001; Eddouks et al., 2002; El-Hilaly et al., 2003; Mehdioui \& Kahouadji, 2007; Tahraoui et al., 2007 ; Hseini, 2008; Salhi et al., 2010 ; Bourkhiss et al., 2013; Daoudi et al., 2013; Nassif et al., 2013). To our knowledge, the South of Morocco made a few ethnobotanical studies (Abouri et al., 2012 ; Saadi et al., 2013). By its geographical situation between the Mediterranean Sea in the North, the Atlantic Ocean on the West, and Sahara in the South, and considering altitudinal and climatic variations, Morocco presents a rich and diversified forest ecosystem. The flora of Morocco has more than 7000 species and subspecies among which approximately 800 are aromatic and medicinal plants (Benabid, 2000). The province of

\section{MATERIALS AND METHODS}

Study area: The area of Agadir Ida Ou tanane, which is part of Souss Massa Draa region, extends over a rough surface of 240.000 hectares and is a part of the Arganeary Reserve Biosphere approved by the UNESCO in 1998. This area is limited to the West by the Atlantic Ocean, to the South by Inezgane Ait Melloul's province, to the North by the provinces of Essaouira and Chichaoua and to the East by the province of Taroudant (figure $n^{\circ} 1$ ). It includes the urban district of Agadir and 12 rural districts of Drarga, Amskroud, Idmine, Tiqqi, Imouzzer, Akesri, Aourir, Taghazout, Tamri, Imsouane, Aziar and Tadrart. The population of Agadir Ida Ou Tanane is about 486048 inhabitants. The density average is about 212 inhabitant $/ \mathrm{km}^{2} .21 .2 \%$ of the population live in rural areas and $78.8 \%$ in urban zones (H.C.P., 2004).
Agadir Ida Ou Tanane is among Morocco regions which possess a potential in medicinal plants (Thyme, lavender, Argan tree). However, this region knows anarchy and intensive use of botanical species exceeding the threshold of bearable regeneration of used resources, which causes a threat for certain species. The valuation of these heritages suffers from a lack of precise knowledge on used species, as well their phytomasse potential and chemical composition. The aim of the present work is to identify medicinal plants species, mode of preparation, part used, handled diseases used by local population of the province of Agadir Ida Ou Tanane and thus to revitalize and to protect local empirical knowledge which stood the test of time (El Rhaffari \& Zaid, 2000). The knowledge of medicinal plants use and procedures applied to their preparation is usually transmitted from generation to generation, but it is often in danger because this transmission is not always assured (Addo-Fordjour et al., 2008; 2012). The inventory of this knowledge is of an extreme utility because it risks disappearing because of the trend of modes of life and means of communication (Bellakhdar, 1997).

The study area can be globally classified in the dry and semi-arid bioclimatic "etage" in freshly hot winter with a seasonal pluviometric regime where more than $51 \%$ of the annual precipitation falls in winter. The area of Ida Ou Tanane is characterized by vegetation particularly formed by a mixture of tropical elements, macaronesian, Mediterranean and endemic (Benabid, 1976). The main plant formations are constituted by the Argan tree, the Thuja, and Holm oak or with a mixture of these species. Agriculture, handicrafts, commerce, and small business establish the skeleton of the rural economy in the mountainous part of Agadir Ida Ou Tanane. These sectors are strengthened at the level of outer-urban municipalities of this province by the employment in the services (Préfecture d'Agadir Ida Ou Tanane, 2011). 


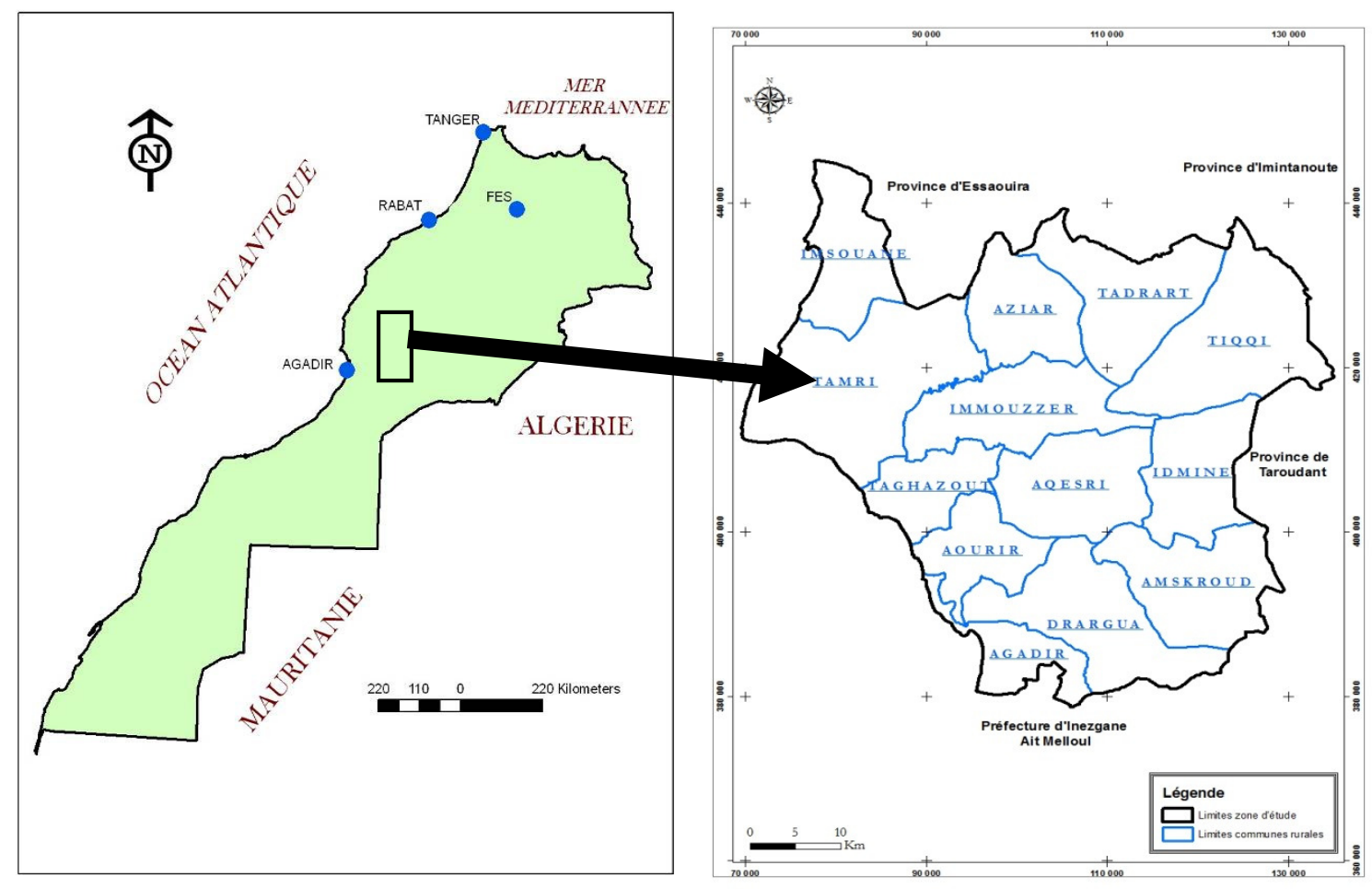

Figure 1: Location of the study area

Data collection: Information was obtained through ethnobotanic inquiries from the inhabitants, and herbal practitioners of the province of Agadir Ida Ou Tanane. The data was collected through semi-structured and structured interviews. To realize this ethnobotanical study, the method of sampling stratified was used to locate the various areas of inquiries (Kahouadji, 1986). Consequently, the zone of study was divided into 13 stratums. Then, samples from 30 to 32 people were investigated in stratum (one urban district: Agadir and twelve rural districts: Drarga, Amskroud, Idmine, Tiqqi, Imouzzer, Akesri, Aourir, Taghazout, Tamri, Imsouane, Aziar and Tadrart). Four hundred (400) interviews were conducted. Collected information concerned the profile of every investigated informant, the choice and the use of plants in traditional medicine, age, sex, level of schooling and income. Then, collected information about every plant used included: its local name (Berber and/or
Arabic), its uses, its origin, its used parts and mode of use or preparation. All the species were mentioned by the respondents by their common label/name. The taxonomic identification of the species was later made by means of the literature: Moroccan Flora' (Fennane et al., 1999, 2007); 'Vascular Flora of Morocco, Inventory and Chorology' (Fennane and Ibn Tattou, 2005, Ibn Tattou and Fennane, 2008), The traditional Moroccan pharmacopoeia (Bellakhdar, 1997), Guide of the healing plants (Pamplona-Roger, 2000), The healing plants of Morocco (Sijelmassi, 2003), Healing plants in the Maghreb and the basic care (Bellakhdar, 2006).

Data analysis: The ethnobotanical data was transferred in a database using software Sphinx and SPSS. The data were analyzed to study the socio-demographic characteristics of informants, diversity of medicinal plants used, therapeutic uses, plant parts used and mode of preparation and administration.

\section{RESULTS AND DISCUSSION}

Interviewee's demographic characteristics: The elderly from 50 to 60 years old have a frequency of use of medicinal plants by $31 \%$ followed by people of more than 60 years of age with a $25 \%$ frequency. The age brackets ( 20 to 30$)$, (31 to 40 ), ( 41 to 50 ) and (<20 years) have a frequency of $7 \%, 13 \%, 22 \%$ and $2 \%$, respectively (figure $n^{\circ} 2$ ). These results show that the older people (more than 50 years with a $56 \%$ frequency) have more knowledge in medicinal plants than the other age groups. This indicates that this kind of knowledge is acquired after a long accumulated experience. The experience accumulated with the age constitutes the main information source on a local scale about the use of plants in 

Province (Southwest Morocco)

traditional medicine (Mehdioui \& Kahouadji, 2007). Most of the informants (91.7 \%) acquired this knowledge from their parents and grandparents. The majority of the inquired subjects (93.7\%) said that they use herbal medicine to handle their disease. A total of 295 individuals depend on herbal medicine only, 78 individuals use herbal medicine before resorting to the modern medicine and 27 people have appeal only to modern medicine.

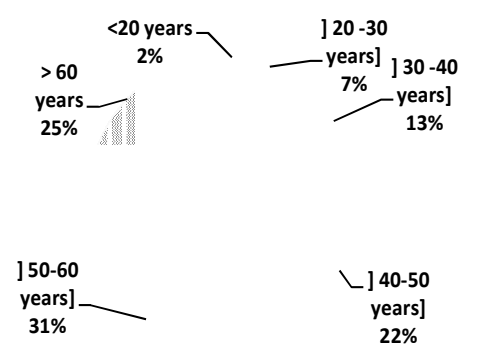

Figure $\mathrm{n}^{\circ} 2$ : Categories of ages

Rural population opt for traditional treatment because its preparation is easy and low cost compared with the modern medicine. This reflects the reality of the local families with low income. Ethno-pharmacological surveys on the use of traditional medicinal materials conducted in other countries revealed similar trends where plants represent most of all the traditional medicinal substances (Addis et al., 2001; Maroyi, 2011). Previous studies have reported a wide range of the rate of plant use (50-95\%), which varied from region to region according to ethnology, richness of medicinal plant sector, and home environment (Sijelmassi, 2003; Bellakhdar, 1997; Ziyyat et al., 1997; Hmammouchi, 1999; Jouad et al., 2001; Eddouks et al., 2002; El-Hilaly et al., 2003; Tahraoui et al., 2007). In the study area, not educated occupy the first row in terms of use of medicinal plants with $52 \%$ (figure $n^{\circ} 3$ ). The people having the primary level of education still have an important part (35\%) contrary to those having education level of secondary and university which represent $9 \%$ and $4 \%$, respectively. These results indicate that interviewed people having a high level of education are bowed towards the modern medicine. The level of illiteracy is relatively high at the level of the province of Agadir Ida Ou Tanane and registers a 59, 65 $\%$ rate which is almost equal to the national average which is $60.2 \%$ (HCP, 2004). Consequently, valuation and sustainable management of natural resources and

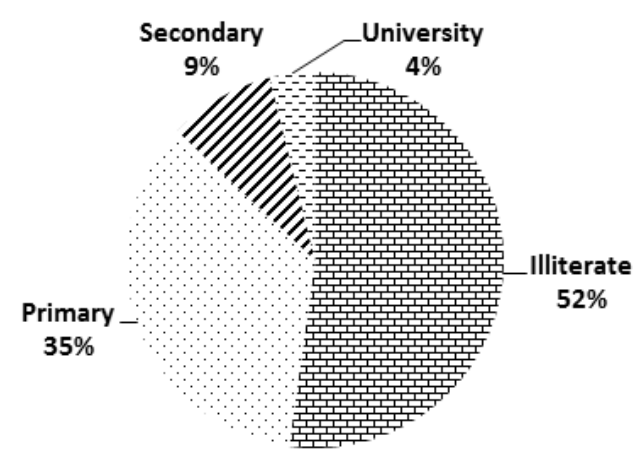

Figure $n^{\circ} 3$ : Educational level

more particularly medicinal plants become difficult. These results are similar to those of other studies in other regions of Morocco (Mehdioui \& Kahouadji, 2007, Abouri et al., 2012, Saadi et al., 2013). The women know better medicinal species than men at the level of the study area (52\% against $48 \%$ respectively). The respondents having a lower income (2500 Dh / month) use medicinal plants more than those having an income more than 2500 Dh / month (65\% against $35 \%$ ); this can be explained by the moderate cost of healing plants. Ethnobotanic studies in other regions of Morocco ended in the same results (Jouad et al., 2001; Eddouks et al., 2002 ; Abouri et al., 2012 ; Saadi et al., 2013).

Diversity of medicinal plants: In this study, we inventoried one hundred and ten plant species belonging to 53 families and 95 genera. The most represented families (figure $\left.n^{\circ} 4\right)$ are Lamiaceae (14 species), Asteraceae (10 species), Apiaceae and Fabaceae (6 species each), Poaceae (4 species) and Anacardiaceae, Brassicaceae, Euphorbiaceae, and Myrtaceae ( 3 species each). The first five families, which are well represented in the study area, exist everywhere in Morocco and constitute the major groups of the medicinal flora in most of other Mediterranean countries (Kahouadji, 1995; Benitez et al., 2010; El Rhaffari \& Zaid, 2000; Savo et al., 2011; Abouri et al., 2012; Nassif \& Tanji, 2013; Saadi et al., 2013). 
Ouhaddou et al.. J. Appl. Biosci. An Ethnobotanical Study of Medicinal Plants of the Agadir Ida Ou Tanane Province (Southwest Morocco)

Table 1: List of medicinal plants used in traditional therapy in Agadir Ida Ou Tanane Province

\begin{tabular}{|c|c|c|c|c|c|}
\hline Scientific name and Family & Local name & Uses & $\begin{array}{l}\text { Plant part } \\
\text { used }\end{array}$ & Preparation & Administration \\
\hline $\begin{array}{l}\text { Ajuga iva (L.) Shreb } \\
\text { Lamiaceae }\end{array}$ & $\begin{array}{l}\text { Timnra Iznkad, } \\
\text { Chendgoura }\end{array}$ & Digestive & $\begin{array}{l}\text { Leaf, root, } \\
\text { seed }\end{array}$ & $\begin{array}{l}\text { Infusion, } \\
\text { powder }\end{array}$ & Oral \\
\hline $\begin{array}{l}\text { Allium cepa L. } \\
\text { Alliaceae }\end{array}$ & Azalim, Basla & Digestive, respiratory & Bulb & $\begin{array}{l}\text { Cataplasm, } \\
\text { decoction }\end{array}$ & Eye drops, oral \\
\hline $\begin{array}{l}\text { Allium sativum L. } \\
\text { Alliaceae }\end{array}$ & Tskert, Touma & $\begin{array}{l}\text { Respiratory, digestive, skin, } \\
\text { skeleton, circulatory }\end{array}$ & Bulb, stem & Decoction & Oral \\
\hline $\begin{array}{l}\text { Aloysia citriodora Palau } \\
\text { Verbenaceae }\end{array}$ & Louiza & $\begin{array}{l}\text { Respiratory, digestive, skin, } \\
\text { skeleton, circulatory, } \\
\text { genital, nervous, auditory, } \\
\text { visual, urinary }\end{array}$ & Leaf & $\begin{array}{l}\text { Infusion, } \\
\text { decoction }\end{array}$ & Oral \\
\hline $\begin{array}{l}\text { Alpinia offinarum Hance } \\
\text { Zingiberaceae }\end{array}$ & Khoudanjal & Skeleton & Root & $\begin{array}{l}\text { Decoction, } \\
\text { powder }\end{array}$ & Oral \\
\hline $\begin{array}{l}\text { Ammodaucus leucotrichus } \\
\text { Coss. \& Durieu } \\
\text { Apiaceae }\end{array}$ & Kamoun sofi & $\begin{array}{l}\text { Digestive, circulatory, } \\
\text { auditory }\end{array}$ & Leaf, seed & Powder & Oral \\
\hline $\begin{array}{l}\text { Apteranthes europea (Guss.) } \\
\text { Merb. } \\
\text { Asclepiadaceae }\end{array}$ & $\begin{array}{l}\text { lgougan, } \\
\text { Oukan }\end{array}$ & Respiratory & Aerial parts & $\begin{array}{l}\text { Decoction, } \\
\text { powder }\end{array}$ & Oral \\
\hline $\begin{array}{l}\text { Argania spinosa (L.) Skeels } \\
\text { Sapotaceae }\end{array}$ & Argan & $\begin{array}{l}\text { Digestive, skin, skeleton, } \\
\text { circulatory, urinary }\end{array}$ & $\begin{array}{l}\text { Leaf, flower, } \\
\text { seed, bark }\end{array}$ & $\begin{array}{l}\text { Infusion, } \\
\text { friction, } \\
\text { powder }\end{array}$ & $\begin{array}{l}\text { Oral, external } \\
\text { use }\end{array}$ \\
\hline $\begin{array}{l}\text { Aristolochia baetica L. } \\
\text { Aristolochiaceae }\end{array}$ & $\begin{array}{l}\text { Azlak ouchen, } \\
\text { Berztem }\end{array}$ & Respiratory & $\begin{array}{l}\text { Leaf, stem, } \\
\text { root, fruit }\end{array}$ & Powder & External use \\
\hline $\begin{array}{l}\text { Artemisia absinthium L. } \\
\text { Asteraceae }\end{array}$ & Chiba & Genital, auditory & Leaf & $\begin{array}{l}\text { Decoction, } \\
\text { powder, } \\
\text { infusion } \\
\end{array}$ & Oral \\
\hline $\begin{array}{l}\text { Artemisia inculta Delile } \\
\text { Asteraceae }\end{array}$ & Izri, Chih & $\begin{array}{l}\text { Respiratory, digestive, skin, } \\
\text { skeleton, circulatory }\end{array}$ & $\begin{array}{l}\text { Leaf, seed, } \\
\text { whole plant }\end{array}$ & \begin{tabular}{|l} 
Decoction, \\
infusion, \\
powder, \\
friction \\
\end{tabular} & Oral \\
\hline $\begin{array}{l}\text { Asparagus altissimus Munby } \\
\text { Asparagaceae }\end{array}$ & Tikrargan & Nervous & Leaf & Powder & Oral \\
\hline $\begin{array}{l}\text { Asparagus offininalis L. } \\
\text { Asparagaceae }\end{array}$ & $\begin{array}{l}\text { Azzwi,Azzû, } \\
\text { Ssekkum }\end{array}$ & Respiratory, nervous & Leaf, stem & Decoction & Oral \\
\hline $\begin{array}{l}\text { Asphodelus ramosus L. } \\
\text { Asphodelaceae }\end{array}$ & Iguri, L-berwag & Skin & Seed, root & $\begin{array}{l}\text { Friction, } \\
\text { mixed with } \\
\text { oil }\end{array}$ & $\begin{array}{l}\text { Applied } \\
\text { externally }\end{array}$ \\
\hline $\begin{array}{l}\text { Asphodelus tenuifolius Cav. } \\
\text { Asphodelaceae }\end{array}$ & Iguri, L-berwag & Digestive, circulatory & Root, fruit & Decoction & Oral \\
\hline $\begin{array}{l}\text { Boswellia sacra Flueck. } \\
\text { Burseraceae }\end{array}$ & $\begin{array}{l}\text { Salaban, } \\
\text { Luban }\end{array}$ & Respiratory & Root & Fumigation & $\begin{array}{l}\text { Inhalation, } \\
\text { external use }\end{array}$ \\
\hline $\begin{array}{l}\text { Brassica rapa L. } \\
\text { Brassicaceae }\end{array}$ & Laft baldi & Digestive & Seed & $\begin{array}{l}\text { Powder } \\
\text { mixed with } \\
\text { honey } \\
\end{array}$ & Oral \\
\hline $\begin{array}{l}\text { Capparis spinosa L } \\
\text { Capparaceae }\end{array}$ & $\begin{array}{l}\text { Tailloloute, } \\
\text { Kabbar }\end{array}$ & Skeleton, nervous & $\begin{array}{l}\text { Root, leaf, } \\
\text { seed }\end{array}$ & $\begin{array}{l}\text { Decoction, } \\
\text { cataplasm }\end{array}$ & $\begin{array}{l}\text { Oral, external } \\
\text { use }\end{array}$ \\
\hline
\end{tabular}


Ouhaddou et al.. J. Appl. Biosci. An Ethnobotanical Study of Medicinal Plants of the Agadir Ida Ou Tanane Province (Southwest Morocco)

\begin{tabular}{l|l|l|l|l|l}
\hline $\begin{array}{l}\text { Carlina gummifera (L.) Less. } \\
\text { Asteraceae }\end{array}$ & Addad, Ahfyun & Circulatory, genital, skin & Root & $\begin{array}{l}\text { Decoction, } \\
\text { cataplasm, } \\
\text { friction }\end{array}$ & $\begin{array}{l}\text { Oral, external } \\
\text { use }\end{array}$ \\
\hline Table 1. continued
\end{tabular}

\begin{tabular}{|c|c|c|c|c|c|}
\hline $\begin{array}{l}\text { Carum carvi L. } \\
\text { Apiaceae }\end{array}$ & Carwiya & Digestive, circulatory & $\begin{array}{l}\text { Stem, seed, } \\
\text { whole plant }\end{array}$ & $\begin{array}{l}\text { Decoction, } \\
\text { powder, } \\
\text { infusion }\end{array}$ & Oral \\
\hline $\begin{array}{l}\text { Chenopodium ambrosioides } \\
\text { L. Chenopodiaceae }\end{array}$ & Mkhinza & $\begin{array}{l}\text { Respiratory, skeleton, } \\
\text { circulatory, nervous }\end{array}$ & $\begin{array}{l}\text { Leaf, whole } \\
\text { plant }\end{array}$ & $\begin{array}{l}\text { Decoction, } \\
\text { powder, } \\
\text { infusion, }\end{array}$ & $\begin{array}{l}\text { External use, } \\
\text { oral }\end{array}$ \\
\hline $\begin{array}{l}\text { Carlina racemosa } \mathrm{L} . \\
\text { Asteraceae }\end{array}$ & Achekja, & Respiratory, digestive, skin & Leaf, root & $\begin{array}{l}\text { Decoction, } \\
\text { infusion }\end{array}$ & $\begin{array}{l}\text { Oral, external } \\
\text { use }\end{array}$ \\
\hline $\begin{array}{l}\text { Ceratonia siliqua L. } \\
\text { Fabaceae }\end{array}$ & $\begin{array}{l}\text { Tikida, } \\
\text { Kharroub }\end{array}$ & Digestive, skin, nervous & $\begin{array}{l}\text { Seed, leaf, } \\
\text { fruit }\end{array}$ & $\begin{array}{l}\text { Infusion, } \\
\text { powder, } \\
\text { decoction }\end{array}$ & Oral \\
\hline $\begin{array}{l}\text { Chamaerops humilis L. } \\
\text { Arecaceae }\end{array}$ & Tiznirt, Doum & Digestive & Seed & Raw & Oral \\
\hline $\begin{array}{l}\text { Cinnamomum verum J. } \\
\text { Presl Lauraceae }\end{array}$ & Lkarfa & Digestive, genital & Stem & $\begin{array}{l}\text { Decoction, } \\
\text { powder }\end{array}$ & Oral \\
\hline $\begin{array}{l}\text { Cistus creticus L. } \\
\text { Cistaceae }\end{array}$ & Irguel & $\begin{array}{l}\text { Respiratory, digestive, } \\
\text { skeleton, circulatory, } \\
\text { urinary }\end{array}$ & $\begin{array}{l}\text { Leaf, seed, } \\
\text { fruit, root, } \\
\text { whole plant }\end{array}$ & $\begin{array}{l}\text { Decoction, } \\
\text { infusion, } \\
\text { powder }\end{array}$ & Oral \\
\hline $\begin{array}{l}\text { Cistus salviifolius L. } \\
\text { Cistaceae }\end{array}$ & Tirguelt & Respiratory, digestive & $\begin{array}{l}\text { Leaf, seed, } \\
\text { stem }\end{array}$ & $\begin{array}{l}\text { Decoction, } \\
\text { infusion, } \\
\text { powder }\end{array}$ & Oral \\
\hline $\begin{array}{l}\text { Citrullus colocynthis (L.) } \\
\text { Shrad. Cucurbitaceae }\end{array}$ & Aferziz, Hadja & $\begin{array}{l}\text { Respiratory, digestive, } \\
\text { skeleton, circulatory }\end{array}$ & $\begin{array}{l}\text { Fruit, whole } \\
\text { plant, pulp }\end{array}$ & $\begin{array}{l}\text { Cataplasm, } \\
\text { infusion, } \\
\text { decoction }\end{array}$ & $\begin{array}{l}\text { Oral, external } \\
\text { use }\end{array}$ \\
\hline $\begin{array}{l}\text { Cladanthus mixtus (L.) } \\
\text { Chevall. Asteraceae }\end{array}$ & $\begin{array}{l}\text { ljdig nbamlal, } \\
\text { Babonj }\end{array}$ & Digestive, skin, circulatory & Capitulum & Friction & $\begin{array}{l}\text { Applied on the } \\
\text { affected part }\end{array}$ \\
\hline $\begin{array}{l}\text { Coriandrum sativum L. } \\
\text { Apiaceae }\end{array}$ & Ikzbour & Digestive & $\begin{array}{l}\text { Seed, aerial } \\
\text { parts }\end{array}$ & $\begin{array}{l}\text { Decoction, } \\
\text { infusion }\end{array}$ & Oral \\
\hline $\begin{array}{l}\text { Crocus sativus L. } \\
\text { Iridaceae }\end{array}$ & Zaâfran & Circulatory, genital & Stigma & Infusion & Oral \\
\hline $\begin{array}{l}\text { Cuminum cyminum } \mathrm{L} \text {. } \\
\text { Apiaceae }\end{array}$ & Lcamon & Digestive, circulatory & Fruit, leaf & $\begin{array}{l}\text { Infusion, } \\
\text { powder }\end{array}$ & $\begin{array}{l}\text { Oral, external } \\
\text { use }\end{array}$ \\
\hline $\begin{array}{l}\text { Cymbopogon schoenanthus } \\
\text { (L.) Spreng. } \\
\text { Poaceae }\end{array}$ & $\begin{array}{l}\text { Tibremt,Tben } \\
\text { mekkâ }\end{array}$ & Skeleton, urinary & $\begin{array}{l}\text { Leaf, whole } \\
\text { plant }\end{array}$ & Infusion & Oral \\
\hline $\begin{array}{l}\text { Cynara scolymus L. } \\
\text { Asteraceae }\end{array}$ & Kharchouf & Respiratory & Root & Decoction & Oral \\
\hline $\begin{array}{l}\text { Dittrichia viscosa (L.) Greuter } \\
\text { Asteraceae }\end{array}$ & $\begin{array}{l}\text { Tinirane, } \\
\text { Terhala }\end{array}$ & Skeleton & Root, leaf & $\begin{array}{l}\text { Powder, } \\
\text { decoction }\end{array}$ & $\begin{array}{l}\text { Oral, external } \\
\text { use }\end{array}$ \\
\hline $\begin{array}{l}\text { Drimia maritima (L.) Stearn } \\
\text { Asparagaceae }\end{array}$ & $\begin{array}{l}\text { Azalim } \\
\text { Ouchen, Bsel } \\
\text { al khenzir }\end{array}$ & Skeleton & Bulb & $\begin{array}{l}\text { Decoction, } \\
\text { maceration }\end{array}$ & $\begin{array}{l}\text { Oral, external } \\
\text { use, inhalation }\end{array}$ \\
\hline
\end{tabular}


Ouhaddou et al.. J. Appl. Biosci. An Ethnobotanical Study of Medicinal Plants of the Agadir Ida Ou Tanane Province (Southwest Morocco)

\begin{tabular}{|c|c|c|c|c|c|}
\hline $\begin{array}{l}\text { Drimia noctiflora (Batt. \& } \\
\text { Trab.) } \\
\text { Asparagaceae }\end{array}$ & $\begin{array}{l}\text { Azalim } \\
\text { ouchen, Bsel } \\
\text { Dib }\end{array}$ & Circulatory & Bulb & $\begin{array}{l}\text { Cataplasm, } \\
\text { maceration }\end{array}$ & $\begin{array}{l}\text { Oral, applied on } \\
\text { the affected } \\
\text { part }\end{array}$ \\
\hline $\begin{array}{l}\text { Echium horridum Batt. } \\
\text { Boraginaceae }\end{array}$ & Tanasat & Urinary & Leaf & Decoction & Oral \\
\hline $\begin{array}{l}\text { Eucalyptus sp. } \\
\text { Myrtaceae }\end{array}$ & $\begin{array}{l}\text { Calitous, } \\
\text { Caliptous }\end{array}$ & Respiratory & Leaf & $\begin{array}{l}\text { Decoction, } \\
\text { infusion, } \\
\text { fumigation }\end{array}$ & $\begin{array}{l}\text { Oral, inhalation, } \\
\text { external use }\end{array}$ \\
\hline $\begin{array}{l}\text { Euphorbia officinarum subsp. } \\
\text { echinus (Hook. f. \& Coss.) } \\
\text { Vindt Euphorbiaceae }\end{array}$ & $\begin{array}{l}\text { Tikiout, } \\
\text { Zaggoum, } \\
\text { Daghmus }\end{array}$ & $\begin{array}{l}\text { Respiratory, skeleton, } \\
\text { circulatory }\end{array}$ & Aerial parts & Powder & Oral \\
\hline \multicolumn{6}{|l|}{ Table 1. continued } \\
\hline $\begin{array}{l}\text { Euphorbia officinarum L. } \\
\text { subsp. officinarum } \\
\text { Euphorbiaceae }\end{array}$ & $\begin{array}{l}\text { Tikiout, } \\
\text { Zaggoum, } \\
\text { Daghmus } \\
\end{array}$ & Respiratory & $\begin{array}{l}\text { Aerial parts, } \\
\text { latex }\end{array}$ & Decoction & $\begin{array}{l}\text { External use, } \\
\text { oral }\end{array}$ \\
\hline $\begin{array}{l}\text { Fagonia cretica L. } \\
\text { Zygophyllaceae }\end{array}$ & Timechkla & Digestive & Whole plant & $\begin{array}{l}\text { Infusion, } \\
\text { powder }\end{array}$ & Oral \\
\hline $\begin{array}{l}\text { Foeniculum vulgare var. } \\
\text { dulce Mill. } \\
\text { Apiaceae }\end{array}$ & Nafaâ, Basbas & Digestive, circulatory & $\begin{array}{l}\text { Fruit, bulb, } \\
\text { seed, whole } \\
\text { plant }\end{array}$ & $\begin{array}{l}\text { Infusion, } \\
\text { decoction, } \\
\text { raw }\end{array}$ & Oral \\
\hline $\begin{array}{l}\text { Genista ifniensis A. } \\
\text { Caballero. Fabaceae }\end{array}$ & Azi, Ouchfoud & Skin & Leaf & $\begin{array}{l}\text { Infusion, } \\
\text { powder }\end{array}$ & External use \\
\hline $\begin{array}{l}\text { Globularia alypum L. } \\
\text { Plantaginaceae }\end{array}$ & $\begin{array}{l}\text { Tasselgha, Ain } \\
\text { arnab }\end{array}$ & $\begin{array}{l}\text { Respiratory, digestive, skin, } \\
\text { circulatory, nervous, urinary }\end{array}$ & $\begin{array}{l}\text { Leaf, stem, } \\
\text { whole plant }\end{array}$ & $\begin{array}{l}\text { Infusion, } \\
\text { decoction, } \\
\text { powder, } \\
\text { friction }\end{array}$ & $\begin{array}{l}\text { Oral, external } \\
\text { use }\end{array}$ \\
\hline $\begin{array}{l}\text { Herniaria cinerea D.C. } \\
\text { Caryophyllaceae }\end{array}$ & Harrast lahjar & Urinary & $\begin{array}{l}\text { Leaf, whole } \\
\text { plant }\end{array}$ & $\begin{array}{l}\text { Decoction, } \\
\text { infusion }\end{array}$ & Oral \\
\hline $\begin{array}{l}\text { Hordeum vulgare L. } \\
\text { Poaceae }\end{array}$ & $\begin{array}{l}\text { Toumzine, } \\
\text { Chaâir }\end{array}$ & Digestive & Seed & Decoction & Oral \\
\hline $\begin{array}{l}\text { Illicium verum Hook. f. } \\
\text { Scisandraceae }\end{array}$ & L-badiane & Digestive & Fruit & Infusion & Oral \\
\hline $\begin{array}{l}\text { Juncus rigidus Desf. } \\
\text { Juncaceae }\end{array}$ & Azmay, Smar & $\begin{array}{l}\text { Skin, circulatory, nervous, } \\
\text { urinary }\end{array}$ & $\begin{array}{l}\text { Leaf, stem, } \\
\text { fruit, seed }\end{array}$ & $\begin{array}{l}\text { Decoction, } \\
\text { infusion }\end{array}$ & Oral \\
\hline $\begin{array}{l}\text { Juniperus oxycedrus L. } \\
\text { Cupressaceae }\end{array}$ & Katran & Skin & Fruit, strain & Decoction & $\begin{array}{l}\text { External use, } \\
\text { inhalation }\end{array}$ \\
\hline $\begin{array}{l}\text { Kleinia anteuphorbium (L.) } \\
\text { Haw. } \\
\text { Asteraceae }\end{array}$ & Acheberdeau & $\begin{array}{l}\text { Respiratory, skeleton, } \\
\text { circulatory }\end{array}$ & Stem & $\begin{array}{l}\text { Juice, } \\
\text { powder, } \\
\text { infusion } \\
\end{array}$ & External use \\
\hline $\begin{array}{l}\text { Launaea arborescens (Batt.) } \\
\text { Murb. } \\
\text { Asteraceae }\end{array}$ & $\begin{array}{l}\text { Ifrskel, Oujan, } \\
\text { Mmu-lbeyna }\end{array}$ & $\begin{array}{l}\text { Respiratory, digestive, skin, } \\
\text { skeleton, circulatory, } \\
\text { urinary }\end{array}$ & Latex, root & $\begin{array}{l}\text { Decoction, } \\
\text { infusion, } \\
\text { powder }\end{array}$ & $\begin{array}{l}\text { Oral, external } \\
\text { use }\end{array}$ \\
\hline $\begin{array}{l}\text { Lavandula dentata L. } \\
\text { Lamiaceae }\end{array}$ & Igerch, Halhal & $\begin{array}{l}\text { Respiratory, digestive, } \\
\text { skeleton, circulatory, } \\
\text { genital, nervous, urinary }\end{array}$ & $\begin{array}{l}\text { Leaf, stem, } \\
\text { root, fruit, } \\
\text { seed, whole } \\
\text { plant }\end{array}$ & $\begin{array}{l}\text { Decoction, } \\
\text { infusion, } \\
\text { powder }\end{array}$ & $\begin{array}{l}\text { Oral, external } \\
\text { use }\end{array}$ \\
\hline
\end{tabular}


Ouhaddou et al.. J. Appl. Biosci. An Ethnobotanical Study of Medicinal Plants of the Agadir Ida Ou Tanane Province (Southwest Morocco)

\begin{tabular}{|c|c|c|c|c|c|}
\hline $\begin{array}{l}\text { Lavandula maroccana Murb. } \\
\text { Lamiaceae }\end{array}$ & Iguiz, Khzama & $\begin{array}{l}\text { Respiratory, digestive, skin, } \\
\text { circulatory, genital }\end{array}$ & $\begin{array}{l}\text { Leaf, fruit, } \\
\text { seed }\end{array}$ & $\begin{array}{l}\text { Decoction, } \\
\text { infusion, } \\
\text { powder }\end{array}$ & $\begin{array}{l}\text { Oral, external } \\
\text { use }\end{array}$ \\
\hline $\begin{array}{l}\text { Lavandula multifida L. } \\
\text { Lamiaceae }\end{array}$ & Iguiz, Khzama & $\begin{array}{l}\text { Respiratory, digestive, } \\
\text { genital, nervous }\end{array}$ & $\begin{array}{l}\text { Leaf, flower, } \\
\text { seed }\end{array}$ & $\begin{array}{l}\text { Infusion, } \\
\text { decoction }\end{array}$ & $\begin{array}{l}\text { Oral, external } \\
\text { use }\end{array}$ \\
\hline $\begin{array}{l}\text { Lavandula stoechas L. } \\
\text { Lamiaceae }\end{array}$ & $\begin{array}{l}\text { Tigercht, } \\
\text { Khzama }\end{array}$ & $\begin{array}{l}\text { Respiratory, digestive, skin, } \\
\text { circulatory, genital }\end{array}$ & $\begin{array}{l}\text { Leaf, flower, } \\
\text { seed }\end{array}$ & $\begin{array}{l}\text { Decoction, } \\
\text { infusion, } \\
\text { powder }\end{array}$ & $\begin{array}{l}\text { Oral, external } \\
\text { use }\end{array}$ \\
\hline $\begin{array}{l}\text { Lepidium sativum L. } \\
\text { Brassicaceae }\end{array}$ & Hab rchad & Respiratory, digestive, skin & Seed & $\begin{array}{l}\text { Infusion, } \\
\text { powder }\end{array}$ & $\begin{array}{l}\text { Oral, external } \\
\text { use }\end{array}$ \\
\hline $\begin{array}{l}\text { Linum usitatissimum L. } \\
\text { Linaceae }\end{array}$ & Zriât lktan & Digestive, urinary & Seed & Powder & Oral \\
\hline
\end{tabular}

Table 1. continued

\begin{tabular}{|c|c|c|c|c|c|}
\hline $\begin{array}{l}\text { Lonicera biflora Desf. } \\
\text { Caprifoliaceae }\end{array}$ & Irifi & Respiratory, digestive & Leaf, fruit & $\begin{array}{l}\text { Infusion, } \\
\text { decoction, } \\
\text { powder }\end{array}$ & Oral \\
\hline $\begin{array}{l}\text { Lycium intricatum Boiss. } \\
\text { Solanaceae }\end{array}$ & $\begin{array}{l}\text { Inzerki, } \\
\text { L'gerdeg }\end{array}$ & Digestive & Seed & Decoction & Oral \\
\hline $\begin{array}{l}\text { Malva parviflora L. } \\
\text { Malvaceae }\end{array}$ & Igoudi & Respiratory & Leaf, stem & $\begin{array}{l}\text { Decoction, } \\
\text { cataplasm }\end{array}$ & $\begin{array}{l}\text { Oral, external } \\
\text { use }\end{array}$ \\
\hline $\begin{array}{l}\text { Malva sylvestris L. } \\
\text { Malvaceae }\end{array}$ & $\begin{array}{l}\text { Tibi, Lkhabiza, } \\
\text { Bakoula }\end{array}$ & Respiratory, urinary & Leaf, root & $\begin{array}{l}\text { Decoction, } \\
\text { cataplasm }\end{array}$ & $\begin{array}{l}\text { Oral, external } \\
\text { use }\end{array}$ \\
\hline $\begin{array}{l}\text { Marrubium vulgare L. } \\
\text { Lamiaceae }\end{array}$ & Ifzi, Merout & $\begin{array}{l}\text { Respiratory, digestive, skin, } \\
\text { auditory }\end{array}$ & $\begin{array}{l}\text { Leaf, whole } \\
\text { plant }\end{array}$ & $\begin{array}{l}\text { Infusion, } \\
\text { powder }\end{array}$ & $\begin{array}{l}\text { Oral, external } \\
\text { use }\end{array}$ \\
\hline $\begin{array}{l}\text { Mentha spicata L. } \\
\text { Lamiaceae }\end{array}$ & $\begin{array}{l}\text { Liqamt, } \\
\text { Naanaa }\end{array}$ & Respiratory, skin & $\begin{array}{l}\text { Leaf, aerial } \\
\text { parts }\end{array}$ & $\begin{array}{l}\text { Infusion, } \\
\text { friction, } \\
\text { decoction }\end{array}$ & $\begin{array}{l}\text { Oral, external } \\
\text { use }\end{array}$ \\
\hline $\begin{array}{l}\text { Mentha suaveolens Ehrh. } \\
\text { Lamiaceae }\end{array}$ & Timija, Mersita & Respiratory, digestive & $\begin{array}{l}\text { Leaf, whole } \\
\text { plant }\end{array}$ & $\begin{array}{l}\text { Infusion, } \\
\text { powder, } \\
\text { decoction }\end{array}$ & Oral \\
\hline $\begin{array}{l}\text { Musa sp. } \\
\text { Musaceae }\end{array}$ & Banane & Skin & Bark & Friction & External use \\
\hline $\begin{array}{l}\text { Myristica fragrans Houtt. } \\
\text { Myristcaceae }\end{array}$ & Lgoza & $\begin{array}{l}\text { Respiratory, digestive, } \\
\text { skeleton, genital }\end{array}$ & Seed & Powder & Oral \\
\hline $\begin{array}{l}\text { Myrtus communis L. } \\
\text { Myrtaceae }\end{array}$ & Rayhan & Digestive, skin & Leaf & $\begin{array}{l}\text { Cataplasm, } \\
\text { decoction, } \\
\text { infusion }\end{array}$ & External use \\
\hline $\begin{array}{l}\text { Nerium oleander L. } \\
\text { Apocynaceae }\end{array}$ & Alili, Dafla & $\begin{array}{l}\text { Skin, nervous, auditory, } \\
\text { visual }\end{array}$ & Leaf, latex & $\begin{array}{l}\text { Powder, } \\
\text { infusion }\end{array}$ & $\begin{array}{l}\text { Oral, inhalation, } \\
\text { external use }\end{array}$ \\
\hline $\begin{array}{l}\text { Nigella sativa L. } \\
\text { Ranunculaceae }\end{array}$ & $\begin{array}{l}\text { Sanouj, } \\
\text { Habba saouda }\end{array}$ & $\begin{array}{l}\text { Respiratory, digestive, } \\
\text { circulatory, visual, urinary }\end{array}$ & Seed & $\begin{array}{l}\text { Powder, } \\
\text { powder } \\
\text { mixed with } \\
\text { honey }\end{array}$ & Oral \\
\hline $\begin{array}{l}\text { Olea europaea L. } \\
\text { Oleaceae }\end{array}$ & $\begin{array}{l}\text { Zitoun, Zit, } \\
\text { Zbouj, } \\
\text { Azemmour }\end{array}$ & $\begin{array}{l}\text { Respiratory, digestive, } \\
\text { circulatory, auditory, } \\
\text { urinary }\end{array}$ & Leaf, fruit & $\begin{array}{l}\text { Decoction, } \\
\text { oil, infusion }\end{array}$ & $\begin{array}{l}\text { Oral, external } \\
\text { use }\end{array}$ \\
\hline
\end{tabular}


Ouhaddou et al.. J. Appl. Biosci. An Ethnobotanical Study of Medicinal Plants of the Agadir Ida Ou Tanane Province (Southwest Morocco)

\begin{tabular}{l|l|l|l|l|l}
\hline $\begin{array}{l}\text { Ononis natrix L. } \\
\text { Fabaceae }\end{array}$ & $\begin{array}{l}\text { Afezdad, } \\
\text { Lhaanna }\end{array}$ & $\begin{array}{l}\text { Respiratory, digestive, } \\
\text { circulatory }\end{array}$ & $\begin{array}{l}\text { Leaf, root, } \\
\text { whole plant }\end{array}$ & $\begin{array}{l}\text { Decoction, } \\
\text { powder, } \\
\text { infusion }\end{array}$ & Oral \\
\hline $\begin{array}{l}\text { Opuntia ficus-indica (L.) Mill. } \\
\text { Cactaceae }\end{array}$ & $\begin{array}{l}\text { Acnari, } \\
\text { Sabbar, } \\
\text { Handiya, } \\
\text { Karmouss } \\
\text { nsara }\end{array}$ & $\begin{array}{l}\text { Respiratory, digestive, } \\
\text { skeleton, circulatory, } \\
\text { urinary }\end{array}$ & Flower, fruit & $\begin{array}{l}\text { Decoction, } \\
\text { powder, } \\
\text { infusion }\end{array}$ & $\begin{array}{l}\text { Oral, external } \\
\text { use }\end{array}$ \\
\hline $\begin{array}{l}\text { Papaver rhoeas L. } \\
\text { Papaveraceae }\end{array}$ & $\begin{array}{l}\text { Kawch, } \\
\text { Bellaaman }\end{array}$ & Skin & Petal, seed & $\begin{array}{l}\text { Decoction, } \\
\text { infusion }\end{array}$ & $\begin{array}{l}\text { Oral, external } \\
\text { use }\end{array}$ \\
\hline $\begin{array}{l}\text { Peganum harmala L. } \\
\text { Nitrariaceae }\end{array}$ & Lharmel & Respiratory, digestive, \\
genital, nervous & Seed, leaf & Fumigation & $\begin{array}{l}\text { Oral, external } \\
\text { use }\end{array}$ \\
\hline $\begin{array}{l}\text { Pennisetum typhoides } \\
\text { Poacm..f.) Stapf \& C.E. Hubb. }\end{array}$ & Illan & Skeleton, genital & Seed & Decoction & Oral \\
\hline $\begin{array}{l}\text { Periploca angustifolia Labill. } \\
\text { Apocynaceae }\end{array}$ & Aslif & Nervous, skeleton & Leaf, root & $\begin{array}{l}\text { Cataplasm, } \\
\text { decoction }\end{array}$ & External use \\
\hline
\end{tabular}

Table 1. continued

\begin{tabular}{|c|c|c|c|c|c|}
\hline $\begin{array}{l}\text { Petroselinum crispum Mill. } \\
\text { Fuss Apiaceae }\end{array}$ & Maadnous & Digestive, urinary & $\begin{array}{l}\text { Leaf, whole } \\
\text { plant, seed }\end{array}$ & Decoction & Oral \\
\hline $\begin{array}{l}\text { Phillyrea angustifolia L. } \\
\text { Oleaceae }\end{array}$ & Tamtoutla & Nervous & Leaf & Decoction & External use \\
\hline $\begin{array}{l}\text { Pinus halepensis Mill. } \\
\text { Pinaceae }\end{array}$ & $\begin{array}{l}\text { Iguenguem, } \\
\text { Sanawbar } \\
\text { (Tayda) }\end{array}$ & $\begin{array}{l}\text { Respiratory, digestive, skin, } \\
\text { circulatory, genital }\end{array}$ & Bark, resin & Powder & $\begin{array}{l}\text { Oral, external } \\
\text { use }\end{array}$ \\
\hline $\begin{array}{l}\text { Pistacia atlantica Desf. } \\
\text { Anacardiaceae }\end{array}$ & Igg, Lebtam & Digestive & $\begin{array}{l}\text { Gum, leaf, } \\
\text { seed }\end{array}$ & $\begin{array}{l}\text { Decoction, } \\
\text { powder }\end{array}$ & Oral \\
\hline $\begin{array}{l}\text { Pistacia lentiscus L. } \\
\text { Anacardiaceae }\end{array}$ & Titkt, Drou & $\begin{array}{l}\text { Respiratory, digestive, skin, } \\
\text { skeleton, circulatory, } \\
\text { nervous, visual, urinary }\end{array}$ & $\begin{array}{l}\text { Leaf, root, } \\
\text { seed, bark }\end{array}$ & $\begin{array}{l}\text { Decoction, } \\
\text { powder, } \\
\text { infusion } \\
\end{array}$ & Oral \\
\hline $\begin{array}{l}\text { Pulicaria mauritanica Batt. } \\
\text { Asteraceae }\end{array}$ & Bamghar & Digestive, circulatory & Flower, leaf & $\begin{array}{l}\text { Decoction, } \\
\text { maceration }\end{array}$ & $\begin{array}{l}\text { Oral, external } \\
\text { use }\end{array}$ \\
\hline $\begin{array}{l}\text { Punica granatum L. } \\
\text { Lythraceae }\end{array}$ & Rmane & Digestive & $\begin{array}{l}\text { Leaf, fruit, } \\
\text { seed, bark }\end{array}$ & $\begin{array}{l}\text { Infusion, } \\
\text { powder, } \\
\text { decoction }\end{array}$ & $\begin{array}{l}\text { Oral, external } \\
\text { use }\end{array}$ \\
\hline $\begin{array}{l}\text { Quercus ilex L. } \\
\text { Fagaceae }\end{array}$ & $\begin{array}{l}\text { Tassaft, } \\
\text { Karrouche, } \\
\text { Ballout } \\
\end{array}$ & $\begin{array}{l}\text { Respiratory, digestive, skin, } \\
\text { skeleton, circulatory }\end{array}$ & $\begin{array}{l}\text { Bark, leaf, } \\
\text { fruit }\end{array}$ & $\begin{array}{l}\text { Infusion, } \\
\text { powder, } \\
\text { decoction } \\
\end{array}$ & $\begin{array}{l}\text { Oral, external } \\
\text { use }\end{array}$ \\
\hline $\begin{array}{l}\text { Retama monosperma (L.) } \\
\text { Boiss. Fabaceae }\end{array}$ & Ilgui, Rtem & Skin, skeleton & Leaf, fruit & infusion & $\begin{array}{l}\text { Oral, external } \\
\text { use }\end{array}$ \\
\hline $\begin{array}{l}\text { Ricinus communis L. } \\
\text { Euphorbiaceae }\end{array}$ & $\begin{array}{l}\text { Awriyur, } \\
\text { Lkherwaa }\end{array}$ & Digestive, skin & Leaf & $\begin{array}{l}\text { Powder, } \\
\text { decoction }\end{array}$ & Oral, massage \\
\hline $\begin{array}{l}\text { Rhus pentaphylla (Jacq.) } \\
\text { Desf. Anacardiaceae }\end{array}$ & Tazzad, Azad & Digestive & Root & Decoction & Oral \\
\hline $\begin{array}{l}\text { Rosa centifolia L. } \\
\text { Rosaceae }\end{array}$ & Tihfert, Ward & Digestive & Flower & $\begin{array}{l}\text { Powder, } \\
\text { decoction }\end{array}$ & $\begin{array}{l}\text { Applied } \\
\text { externally oral }\end{array}$ \\
\hline
\end{tabular}


Ouhaddou et al.. J. Appl. Biosci. An Ethnobotanical Study of Medicinal Plants of the Agadir Ida Ou Tanane Province (Southwest Morocco)

\begin{tabular}{l|l|l|l|l|l}
\hline $\begin{array}{l}\text { Rosmarinus officinalis L. } \\
\text { Lamiaceae }\end{array}$ & Azir & Respiratory, digestive & $\begin{array}{l}\text { Leaf, aerial } \\
\text { parts }\end{array}$ & $\begin{array}{l}\text { Decoction, } \\
\text { infusion }\end{array}$ & $\begin{array}{l}\text { Oral, external } \\
\text { use }\end{array}$ \\
\hline $\begin{array}{l}\text { Rubia peregrina L. } \\
\text { Rubiaceae }\end{array}$ & Tarouba, Foua & Digestive, circulatory & $\begin{array}{l}\text { Root, whole } \\
\text { plant, leaf, } \\
\text { seed }\end{array}$ & $\begin{array}{l}\text { Powder, } \\
\text { decoction }\end{array}$ & Oral \\
\hline $\begin{array}{l}\text { Rumex acetosa L. } \\
\text { Polygonaceae }\end{array}$ & Tismoumine & Skin & $\begin{array}{l}\text { Seed, leaf, } \\
\text { root }\end{array}$ & $\begin{array}{l}\text { Powder, } \\
\text { decoction }\end{array}$ & Oral \\
\hline $\begin{array}{l}\text { Ruta montana (L.) L. } \\
\text { Rutaceae }\end{array}$ & L-fijel, Awermi & $\begin{array}{l}\text { Respiratory, skin, skeleton, } \\
\text { circulatory, auditory }\end{array}$ & Whole plant & $\begin{array}{l}\text { Friction, } \\
\text { infusion }\end{array}$ & $\begin{array}{l}\text { External use, } \\
\text { oral }\end{array}$ \\
\hline $\begin{array}{l}\text { Salvia aegyptiaca L. } \\
\text { Lamiaceae }\end{array}$ & Iderki & Respiratory, circulatory & $\begin{array}{l}\text { Aerial parts, } \\
\text { root, leaf }\end{array}$ & $\begin{array}{l}\text { Infusion, } \\
\text { decoction }\end{array}$ & Oral \\
\hline $\begin{array}{l}\text { Salvia officinalis L. } \\
\text { Lamiaceae }\end{array}$ & Salmia & $\begin{array}{l}\text { Respiratory, digestive, } \\
\text { circulatory }\end{array}$ & $\begin{array}{l}\text { Leaf, whole } \\
\text { plant }\end{array}$ & $\begin{array}{l}\text { Infusion, } \\
\text { decoction }\end{array}$ & Oral \\
\hline $\begin{array}{l}\text { Senna alexandrina Mill. } \\
\text { Fabaceae }\end{array}$ & Sanae & Digestive & Leaf, seed & Infusion & $\begin{array}{l}\text { Oral, external } \\
\text { use }\end{array}$ \\
\hline $\begin{array}{l}\text { Silene vulgaris (Moench) } \\
\text { Garcke } \\
\text { Caryophyllaceae }\end{array}$ & $\begin{array}{l}\text { Tighercht, } \\
\text { Sabun el-fqar }\end{array}$ & Digestive, skin, genital & Root & $\begin{array}{l}\text { Decoction, } \\
\text { infusion }\end{array}$ & Oral \\
\hline
\end{tabular}

Table 1. continued

\begin{tabular}{|c|c|c|c|c|c|}
\hline $\begin{array}{l}\text { Sinapis alba L. } \\
\text { Brassicaceae }\end{array}$ & $\begin{array}{l}\text { Kalkas, } \\
\text { Akhssur oufkir }\end{array}$ & Circulatory, genital & Seed & $\begin{array}{l}\text { Powder } \\
\text { mixed with } \\
\text { honey, milk, } \\
\text { oil }\end{array}$ & Oral \\
\hline $\begin{array}{l}\text { Syzygium aromaticum (L.) } \\
\text { Merr. \& L. M. Perry } \\
\text { Myrtaceae }\end{array}$ & Korenfal & $\begin{array}{l}\text { Digestive, circulatory, } \\
\text { urinary }\end{array}$ & Clove & $\begin{array}{l}\text { Decoction, } \\
\text { cataplasm }\end{array}$ & $\begin{array}{l}\text { Oral, external } \\
\text { use }\end{array}$ \\
\hline $\begin{array}{l}\text { Tetraclinis articulata (Vahl) } \\
\text { Mast. } \\
\text { Cupressaceae }\end{array}$ & Azouka, Aârar & $\begin{array}{l}\text { Respiratory, digestive, skin, } \\
\text { skeleton, circulatory, } \\
\text { nervous, urinary }\end{array}$ & Leaf, cone & $\begin{array}{l}\text { Cataplasm, } \\
\text { decoction, } \\
\text { powder, } \\
\text { infusion }\end{array}$ & $\begin{array}{l}\text { Oral, external } \\
\text { use }\end{array}$ \\
\hline $\begin{array}{l}\text { Thymus broussonetii Boiss } \\
\text { Lamiaceae }\end{array}$ & $\begin{array}{l}\text { Tazouknnit, } \\
\text { Zaitra }\end{array}$ & $\begin{array}{l}\text { Respiratory, digestive, skin, } \\
\text { circulatory, genital, } \\
\text { nervous, urinary }\end{array}$ & $\begin{array}{l}\text { Leaf, stem, } \\
\text { whole plant }\end{array}$ & $\begin{array}{l}\text { Decoction, } \\
\text { infusion }\end{array}$ & Oral \\
\hline $\begin{array}{l}\text { Thymus pallidus Coss. } \\
\text { ex.Batt. Lamiaceae }\end{array}$ & $\begin{array}{l}\text { Tajllabet, } \\
\text { Ajllab }\end{array}$ & $\begin{array}{l}\text { Respiratory, digestive, } \\
\text { circulatory }\end{array}$ & Flower, leaf & $\begin{array}{l}\text { Decoction, } \\
\text { infusion }\end{array}$ & Oral \\
\hline $\begin{array}{l}\text { Thymus satureioides Coss. } \\
\text { Lamiaceae }\end{array}$ & $\begin{array}{l}\text { Azoukni, } \\
\text { Zaater }\end{array}$ & $\begin{array}{l}\text { Respiratory, digestive, skin, } \\
\text { circulatory, genital, } \\
\text { nervous, visual, urinary }\end{array}$ & $\begin{array}{l}\text { Flower, leaf, } \\
\text { stem, whole } \\
\text { plant }\end{array}$ & Infusion & $\begin{array}{l}\text { Oral, external } \\
\text { use }\end{array}$ \\
\hline $\begin{array}{l}\text { Trigonella foenum-graecum } \\
\text { L. Fabaceae }\end{array}$ & Tifidass, Halba & $\begin{array}{l}\text { Digestive, circulatory, } \\
\text { genital }\end{array}$ & Seed, fruit & $\begin{array}{l}\text { Decoction, } \\
\text { infusion, } \\
\text { powder }\end{array}$ & Oral \\
\hline $\begin{array}{l}\text { Triticum aestivum } \mathrm{L} \\
\text { Poaceae }\end{array}$ & $\begin{array}{l}\text { L-gemh, Irden, } \\
\text { Zraa }\end{array}$ & Digestive & Seed & Powder & Oral \\
\hline $\begin{array}{l}\text { Urtica urens L. } \\
\text { Urticaceae }\end{array}$ & $\begin{array}{l}\text { Azentak, } \\
\text { Harriga }\end{array}$ & Respiratory, urinary & Seed, leaf & $\begin{array}{l}\text { Powder, } \\
\text { decoction, } \\
\text { infusion }\end{array}$ & Oral \\
\hline
\end{tabular}


Ouhaddou et al.. J. Appl. Biosci. An Ethnobotanical Study of Medicinal Plants of the Agadir Ida Ou Tanane Province (Southwest Morocco)

\begin{tabular}{l|l|l|l|l|l}
\hline $\begin{array}{l}\text { Vitex agnus-castus L. } \\
\text { Verbenaceae }\end{array}$ & $\begin{array}{l}\text { Angaref, } \\
\text { Lkherwaa, } \\
\text { Habb el-faqd }\end{array}$ & Skeleton, genital & $\begin{array}{l}\text { Leaf, fruit, } \\
\text { seed }\end{array}$ & $\begin{array}{l}\text { Infusion, } \\
\text { decoction, } \\
\text { powder }\end{array}$ & Oral \\
\hline $\begin{array}{l}\text { Withania frutescens (L.) } \\
\text { Pauquy Solanaceae }\end{array}$ & $\begin{array}{l}\text { Tiremt, } \\
\text { Tarremt }\end{array}$ & Respiratory, skin & $\begin{array}{l}\text { Leaf, bark, } \\
\text { root }\end{array}$ & $\begin{array}{l}\text { Infusion, } \\
\text { powder }\end{array}$ & $\begin{array}{l}\text { Oral, applied on } \\
\text { the affected } \\
\text { part }\end{array}$ \\
\hline $\begin{array}{l}\text { Zingiber officinale Roscoe } \\
\text { Zingiberaceae }\end{array}$ & $\begin{array}{l}\text { Sknjbir, } \\
\text { Zanjabil }\end{array}$ & $\begin{array}{l}\text { Respiratory, digestive, } \\
\text { skeleton, circulatory, } \\
\text { auditory }\end{array}$ & Rhizome & $\begin{array}{l}\text { Decoction, } \\
\text { infusion }\end{array}$ & Oral \\
\hline $\begin{array}{l}\text { Ziziphus lotus (L.) Lam. } \\
\text { Rhamnaceae }\end{array}$ & $\begin{array}{l}\text { Azegar, Sedra, } \\
\text { Nbeg } \\
\text { uigestive, circulatory, }\end{array}$ & $\begin{array}{l}\text { Fruit, leaf, } \\
\text { root }\end{array}$ & $\begin{array}{l}\text { Cataplasm, } \\
\text { infusion, } \\
\text { powder }\end{array}$ & $\begin{array}{l}\text { Oral, external } \\
\text { use }\end{array}$ \\
\hline
\end{tabular}

Most of the identified families are represented by one or two species, which shows that the used healing plants are ot only concentrated in some families and genera. This agrees with other ethnobotanic studies made outside
Morocco and in the Mediterranean region (Raja et al., 1997; Merzouki, 2000; Novais et al., 2004; Tahraoui et al., 2007; Ugulu et al., 2009).

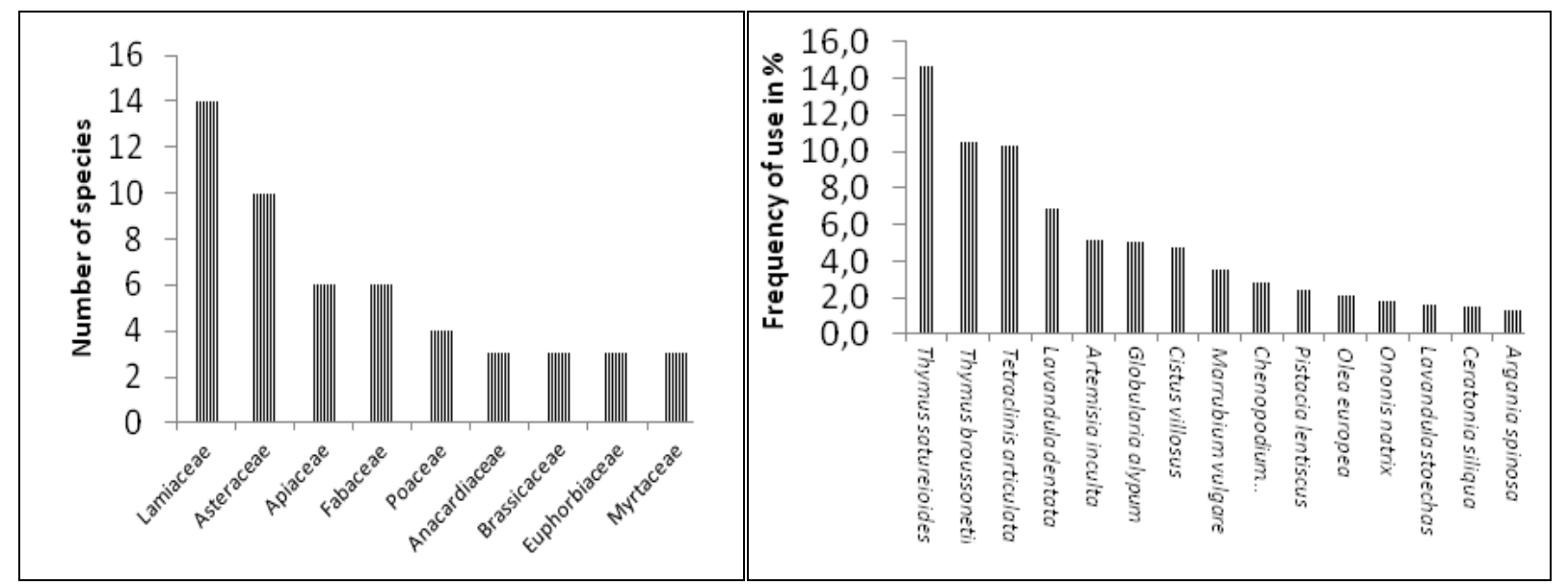

Figure 4: Most represented families

Figure 5: Most used species

Outcomes of the investigation show that 15 medicinal plants are the most used in the study area (figure $n^{\circ} 5$ ): Thymus satureioides (cited 238 times), Thymus broussonetii (171), Tetraclinis articulata (168), Lavandula dentata (111), Artemisia inculta (84), Globularia alypum (83), Cistus villosus (78), Marrubium vulgare (58), Chenopodium ambrosoides (46), Pistacia lentiscus (39), Olea europea (34), Ononis natrix (30), Lavandula stoechas (26), Ceratonia siliqua (25) and Argania spinosa (22). These species are used frequently in other regions of Morocco (Abouri et al., 2012). Of special interest, among the species used at Agadir Ida Ou Tanane province, 8 species $(7.27 \%)$ are endemic of Morocco. The majority of healing plants $(65.45 \%)$ registered in this survey are spontaneous species (Thymus satureioides, Argania spinosa, Tetraclinis articulata, Pistacia lentiscus, Lavandula dentata, and Ononis natrix). Many plants (19.1
$\%$ are cultivated for food purposes (Artemisia absinthium, Coriandrum sativum, Carum carvi, Hordeum vulgare, Mentha viridis). Plants imported from other regions of Morocco or from other countries represent $15.45 \%$ (Crocus sativus, Boswellia sacra, Eugenia caryophyllata, Lepidium sativum and Myristica fragrans). These results are similar to those of other studies carried out in certain zones of Morocco (Abouri et al., 2012; Saadi et al., 2013). The large number of plant species used in the study area indicates the dependence of local population on medicinal plants to handle various diseases and represent a good indicator of a deep knowledge of plants by the local population. The analysis of the biological forms of healing plants used in the study area revealed that most of the species are shrubs (32\%), followed by herbs $(21 \%)$, trees (16\%), hemicryptophytes (12\%), subshrubs (11\%), and geophytes (8\%) (Figure 
$\left.n^{\circ} 6\right)$. This result is against other studies in which trees were the shape of the most dominant growth (AddoFordjour et al., 2008; Moshi et al., 2009). In the study area, as in most regions of Morocco as well as in other countries, a conflict exists between plant use and resources preservation (Sheldon et al., 1998; Kala, 2000; Agelet \& Vallés, 2001). The species of Thymus satureioides and Argania spinosa are subject to a strong pressure of use for medicinal and commercial purposes. This factor combined with an increasing pressure of the local population, besides the irregular precipitation like that of last years, can lead to the reduction of the natural habitat of healing plants.

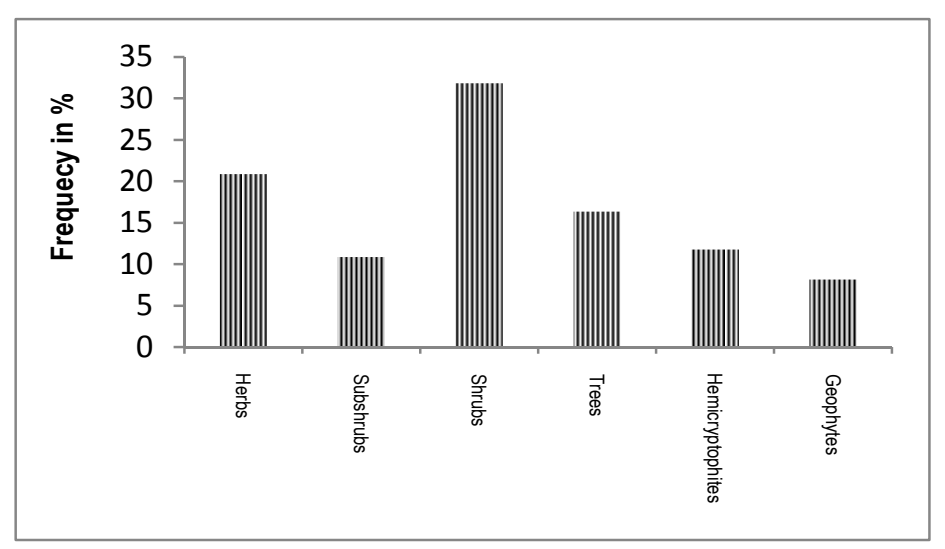

Figure 6: Growth habit of the plants used in Agadir Ida Ou Tanane Province

Therapeutic uses: The species indicated in the table $n^{\circ} 1$ are used by local population of Agadir Ida Ou Tanane to handle various diseases. It was noticed that each of the plants has more than one therapeutic use. For example, Thymus satureioides is used against respiratory and digestive tracts disease; which explains the particular pressure exerted on this species plant in this province. All diseases were structured according to the device or the system which they affect: digestive system, respiratory system, circulatory system, reproductive organ, genito-urinary organs, ear, nose and throat system, ocular system, skin, skeleton and nervous system. The population of the study area suffers; first, from all diseases affecting digestive system (22\%) especially stomach pain, diarrheas, intestinal gases, constipations, etc. Gastro-intestinal disorders are found to be the most common application of medicinal plants by ethnobotanical surveys carried out in other studies (Merzouki et al., 2000; El-Hilaly et al., 2003; Benitez et al., 2010; Mati \& De Boer, 2011). The-diseases of the respiratory system come in the second place with $18 \%$ rate: asthmas, rum, coughs and lung affections. The diseases affecting the circulatory system (low blood pressure, high blood pressure, glycemia) and the skin (cook hair, facial skincare, the eczema, the burns, the wounds) come in the third position with $16 \%$ and $12 \%$ rate respectively. This noticed are similar to those of the study carried out at the level of the province of Rabat (Hseini \& Kahouadji, 2007). The other diseases concern skeleton $(9 \%)$, urinary system $(8 \%)$, reproductive organ and nervous system (6\% each), hearing aid (2\%) and visual device $(1 \%)$.

Plant parts used, mode of preparation and administration : The results obtained at the level of the province of Agadir Ida Ou Tanane show that the most used plant part is the leaf $(74 \%$ ) followed by seed, fruit, root, whole plant, stem and flower with a respective percentage of $8 \%, 5 \%, 4 \%, 3 \%, 2 \%$ and $1 \%$ (figure $\mathrm{n}^{\circ} 7$ ). The underground organs (bulbs and roots) of plants used locally for medicinal purpose represented around 6 $\%$. This percentage is relatively lower to what has been found in other Moroccan regions (Ouarghidi et al., 2013). The high rate of leaves use can be explained by the harvest ease but also, by the fact that these organs are exposed to the sun what gets those virtues and benefactions (El Rhaffari \& Zaid, 2000). This can be explained by the photosynthesis phenomenon which favours the biosynthesis and the storage of metabolites. Also, leaves of plants have been reported to accumulate, inulins, tannins and other alkaloids which may be responsible for their medicinal properties, explaining its wide use (Simbo, 2010). 


\section{Ouhaddou et al.. J. Appl. Biosci. An Ethnobotanical Study of Medicinal Plants of the Agadir Ida Ou Tanane}

Province (Southwest Morocco)

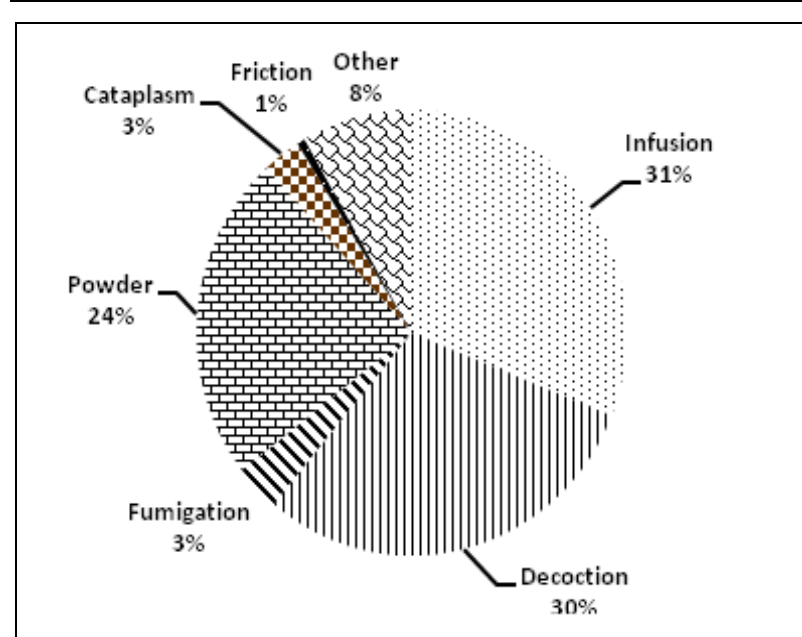

Figure 8: Methods of preparation of plants

The methods used in the zone of study are: the infusion, the decoction, the powder, the fumigation, the cataplasm, the friction, the maceration, plant brute or in mixture with the honey or the oil, etc. The local population always looks for the easiest mode to prepare phytomedicaments. The decoction, the infusion and the powder were generally the method of choice, accounting for 31,30 and $24 \%$ respectively, followed by fumigation preparation $(3 \%)$, cataplasm $(3 \%)$ and friction (1\%) (Figure $\mathrm{n}^{\circ} 8$ ). The decoction allows collecting most active ingredients and limiting or cancels the toxic effect of certain recipes (Salhi, 2010). In other regions of Morocco, decoction and infusion were also the most used herbs preparations (Ziyyat et al., 1997; Merzouki et al., 2000; El-Hilaly et al., 2003; Tahraoui et al., 2007, Abouri et al., 2012). Most preparations were drawn from single plant, but their mixtures are also generally used. Some remedies contain varying combinations of plant organs. For $15 \%$ of plants,

\section{CONCLUSION}

The present study shows that a large number of medicinal plants are used for treating different ailments highlighting significant cultural diversity in the Agadir Ida Ou Tanane province. The elderly and people with no formal education were found the most users of this herbal medicine and consequently are more knowledgeable, which underlies the question on the conservation of this traditional knowledge through generations. Most of the medicinal plants used are sourced from the wild and some of themes are unfortunately over-exploited, which threatened their survival in the area. Digestive disorders were found as the most ailments treated with plants in the

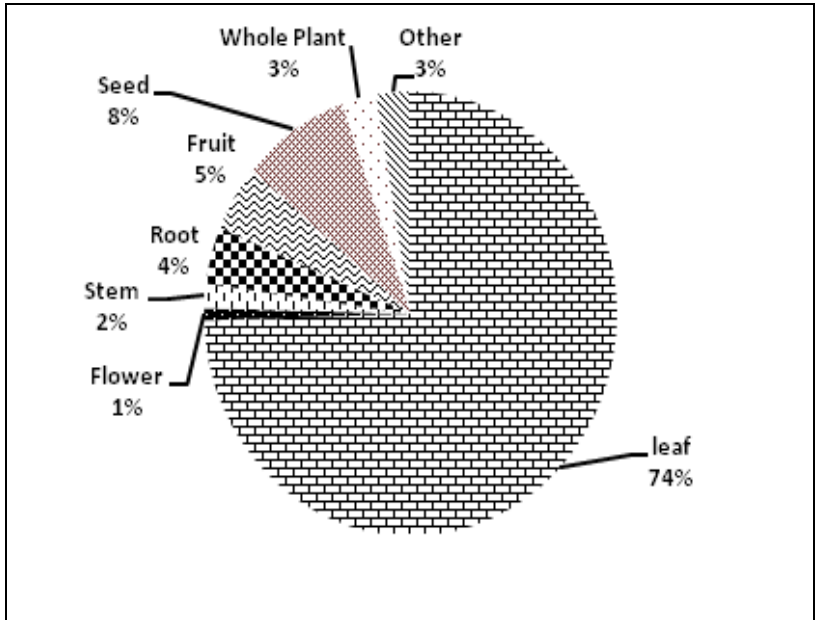

Figure 7: Plants parts used

all plants parts were used. Furthermore, the use of more than a species of plant to prepare a remedy for diseases is attributed to additive or effects of synergy which they could have (Bussman \& Sharon, 2006). Most of the preparations are made with water as a solvent. Diverse parts of the plant were also mixed with oil, honey, milk or tea to improve their acceptability and their medicinal properties. For example, the powder of Nigella sativa mixed with honey is used for respiratory, digestive and urinary system diseases. The mode of administration mostly used is oral $(64.5 \%)$. Herb teas (obtained by infusion or decoction) are usually drunk as teas. The external application was also used with $31.5 \%$ rate and consists, generally, in a local application of the affected part. The remaining modes (eye-drops, wash of mouth) represents $5 \%$ only. These modes of administration are similar to those brought back by Saadi et al.(2013).

province. Decoctions and infusions were the most common method of preparation and most of the medicines are administered orally. It's clear that the documentation of traditional knowledge on medicinal plants in Agadir Ida Ou Tanane province still needs more efforts at the national level, to prevent this valuable knowledge from being lost. Such ethnobotanical studies are essential for this purpose because they contribute to document the therapeutic practices of the community and consequently permit to consolidate the indigenous knowledge of the local population. 


\section{REFERENCES}

Abouri M, El Mousadik A, Msanda F, Boubaker H, Saadi B, Cherifi K,2012. An ethnobotanical survey of medicinal plants used in the Tata Province, Morocco. International Journal of Medicinal Plants Research 1(7): 99-123

Addis $G$, Abebe D, Urga K, 2001. A survey of traditional medicine plants in Shirka District, Arsi Zone, Ethiopia. Ethiopian Pharmaceutical Journal 19: 30-47.

Addo-Fordjour P, Anning AK, Akanwariwiak WG, Belford EJD, Firempong CK, 2012. Medicinal plants of Ghana. In: Singh, R.J. (Ed.) Genetic resources, chromosome engineering, and crop improvement: Medicinal plants. Volume 6. CRC Press, Taylor and Francis Group, LLC, USA, pp. 221-246.

Addo-Fordjour P, Anning AK, Belford EJD, Akonnor D, 2008. Diversity and conservation of medicinal plants in the Bomaa community of the Brong Ahafo region, Ghana. International Journal of Medicinal Plants Research 2(9): 226-233.

Agelet A. and Vallès J, 2001. Studies on pharmaceutical ethnobotany in the region of Pallars (Pyrenees, Catalonia, Iberian Peninsula). Part I. General results and new or very rare medicinal plants. Journal of Ethnopharmacology 77: 57-70.

Al-Adhroey A H, Nor ZM, Al-Mekhlafi HM, Mahmud R, 2010. Ethnobotanical study on some Malaysian anti-malarial plants: A community based survey. Journal of Ethnopharmacology 132: 362-364.

Bellakhdar J, Claisse R, Fleurentin J, Younos C, 1991. Repertory of standard herbal drugs in the Moroccan pharmacopoea. Journal of Ethnopharmacology 35:123-143.

Bellakhdar J, 1997. La pharmacopée marocaine traditionnelle. Médecine arabe ancienne et avoirs populaires. Ibis Press, Paris. 764 pp.

Bellakhdar J, 2006. Plantes médicinales au Maghreb et soins de base. Précis de phytothérapie moderne. Editions le Fennec, Casablanca, Maroc. 386p.

Bellakhdar J, 2006. Plantes médicinales au Maghreb et soins de base. Précis de phytothérapie moderne. Editions le Fennec, Casablanca, Maroc. 386p.

Benabid A, 1976. Etude écologique et phytosociologique et sylvopastorale de la tétraclinaie de l'Amsitten. Thèse doctorat de 3ème cycle, Fac. Sci. St Jérôme Marseille III, France, $155 \mathrm{p}$.
Benabid A, 2000. Flore et écosystèmes du Maroc, Évaluation et préservation de la biodiversité. Ibis Press, Paris, pp. 360.

Benitez G, Gonzalez-Tejero MR, Molero-Mesa J, 2010. Pharmaceutical ethnobotany in the western part of Granada province (southern Spain): Ethnopharmacological synthesis. Journal of Ethnopharmacology 129: 87-105.

Bourkhiss M, Farah A, Satrani B, Lakhlifi T, Rassam A, Bourkhiss B, Chaouch A, 2013. Étude ethnobotanique des plantes médicinales dans la région de Tafilalet, Sud-est marocain, La Phytothérapie Européenne, Mars-Avril 2013 : 25-28

Bussman RW. and Sharon D, 2006. Traditional medicinal plant use in Northern Peru: tracking two thousand years of healing culture. Journal of Ethnobiology and Ethnomedicine, 2: 47.

Daoudi A, Zerkani S, Nassiri L, Boukil M, Ibijbijen J, 2013. Inventaire des plantes médicinales de la commune d'Aguelmouss, province de Khénifra, Maroc, Science Lib Editions Mersenne : Volume $5, N^{\circ} 131012$, ISSN 2111-4706

Eddouks M, Maghrani M, Lemhadri A, Ouahidi ML, Jouad $\mathrm{H}$, 2002. Ethnopharmacological survey of medicinal plants used for the treatment of diabetes mellitus, hypertension and cardiac diseases in the south-east region of Morocco (Tafilalet). Journal of Ethnopharmacology 82: 97-103

Eddouks M, Ouahidi L, Farid O, Moufid A, Khalidi A, Lemhadri A, 2007. Utilisation des plantes médicinales dans le traitement du diabète au Maroc. Phytothérapie 5: 194-203.

El-Hilaly J, Hmammouchi M, Lyoussi B, 2003. Ethnobotanical studies and economic evaluation of medicinal plants in Taounate Province (Northern Morocco). Journal of Ethnopharmacology 86: 149-158.

El Rhaffari U. and Zaid A, 2000. Pratique de la phytothérapie dans le sud-est du Maroc (Tafilalet). Un savoir empirique pour une pharmacopée rénovée. Des sources du savoir aux médicaments du futur. IRD Editions. Actes $\mathrm{du} \quad 4$ ème Congrès européen d'ethnopharmacologie, 11-13 mai 2000, Metz (France).

Fennane M. and Ibn Tattou M, 2005. Flore vasculaire du Maroc. Inventaire et chorologie. Vol. 1. Travaux de l'Institut Scientifique, série botanique, p. 37. 
Fennane M, Ibn Tattou M, Mathez J, Ouyahya A, Oualidi J, 1999. Flore Pratique du Maroc, Vol. 1 : Pteridophyta, Gymnospermae, Angiospermae (Lauraceae-Neuradaceae): Manuel de Détermination. Travaux de l'Institut Scientifique, série botanique p. 36 .

Fennane M, Ibn Tattou M, Mathez J, Ouyahya A, Oualidi J, 2007. Flore Pratique du Maroc, Vol. 2 : Pteridophyta, Gymnospermae, Angiospermae (Lauraceae-Neuradaceae): Manuel de Détermination Travaux de l'Institut Scientifique, série botanique p. 38.

Ghanmi M, Satrani B, Aberchane M, Ismaili M R, Aafi A, El Abid A, 2011. Plantes Aromatiques et Médicinales du Maroc, les milles et une vertu. Centre de Recherche Forestière. Rabat, Maroc $130 \mathrm{p}$.

Haut-Commissariat au Plan (H.C.P.), 2004. Recensement général de la population et de l'habitat. Site Internet http://www.clad.hcp.ma/resultatsdurgph2004/

Hamilton A, 2003. Medicinal Plants and Conservation: Issues and Approaches. Surrey (RoyaumeUni) : International Plants Conservation Unit, WWFUK, 51 pages.

Hmammouchi M, 1999. Les plantes médicinales et aromatiques marocaines. Utilisations, biologie, écologie, chimie, pharmacologie, toxicologie et lexiques. Deuxième édition, Imprimerie Fedala. Rabat-Instituts, $450 \mathrm{pp}$.

Hseini S. and Kahouadji A, 2007. Etude ethnobotanique de la flore médicinale dans la région de Rabat (Maroc occidental), LAZAROA 28 : 79-93.

Hseini S, 2008. Etude ethnobotanique de la flore médicinale dans la Région de Rabat, Thèse de Doctorat, Université Mohamed V- Agdal, Fac. Sci. Rabat. Maroc, $175 \mathrm{pp}$.

Ibn Tattou M. and Fennane M, 2008. Flore vasculaire du Maroc. Inventaire et chorologie. Vol. 2. Travaux de l'Institut Scientifique, série botanique p. 39.

Jouad H, Haloui M, Rhiouani H, El Hilaly J, Eddouks M, 2001. Ethnobotanical survey of medicinal plants used for the treatment of diabetes, cardiac and renal diseases in the North centre region of Morocco (Fez-Boulemane). Journal of Ethnopharmacology 77: 175-182.

Kahouadji S, 1995. Contribution à une étude ethnobotanique des plantes médicinales dans le Maroc oriental, Thèse de Doctorat 3ème cycle, Université Mohamed I, Fac. Sci. Oujda, 220 pp.
Kahouadji A, 1986. Recherches floristiques sur le massif montagneux des Béni Snassène (Maroc oriental). Thèse, Université des Sciences et Techniques du Languedoc. Montpellier, $235 \mathrm{pp}$.

Kala CP, 2000. Status and conservation of rare and endangered medicinal plants in the Indian transHimalaya. Biological Conservation 93: 371-379.

Maroyi A, 2011. An ethnobotanical survey of medicinal plants used by the people in Nehma communal area, Zimbabwe. Journal of Ethnopharmacology 136: 347-354.

Mati E. and De Boer H, 2011. Ethnobotany and trade of medicinal plants in the Qaysari Market, Kurdish Autonomous Region, Iraq. Journal of Ethnopharmacology 133: 490-510.

Mehdioui R. and Kahouadji A, 2007. Etude ethnobotanique dans la région d'Essaouira, Bulletin de l'institut scientifique, Rabat, Maroc, section Sciences de la vie, 29, 11-20.

Merzouki A, Ed-derfoufi F, Molero Mesa J, 2000. Contribution to the knowledge of Rifian traditional medicine. II: Folk medicine in Ksar Lakbir district (NW Morocco). Fitoterapia 71: 278-307

Moshi MJ, Otieno DF, Mbabazi PK, Weisheit A, 2009. The Ethnomedicine of the Haya people of Bugabo ward, Kagera Region, north western Tanzania. Journal of Ethnobiology and Ethnomedicine 5: 24.

Nassif F. and Tanji A, 2013. Gathered food plants in Morocco: the long forgotten species in ethnobotanical research, Life sciences leaflets, pp 17-54.

Novais MH, Santos I, Mendes S, Pinto-Gomes C, 2004. Studies on pharmaceutical ethnobotany in Arrabida Natural Park (Portugal). Journal of Ethnopharmacology 93: 183-195

OMS, UICN \& WWF, 1993. Principes directeurs pour la conservation des plantes médicinales, Gland, Suisse, $35 \mathrm{p}$.

Ouarghidi A, Martin GJ, Powell B, Esser G, Abbad A, 2013. Botanical identification of medicinal roots collected and traded in Morocco and comparison to the existing literature. Journal of Ethnobiology and Ethnomedicine, 9:59

Pamplona-Roger G, 2000. Guide des plantes médicinales. Bibliothèque Education et Santé. Editorial Safeliz (Madrid) et Editions Vie et Santé (Cedex), 796 pp.

Peyron L, 2000. Aspect international actuel du marché des plantes aromatiques et médicinales, actes 
de la journée de réflexion sur les plantes aromatiques et médicinales, casablanca, pp 314.

Préfecture d'Agadir Ida Ou Tanane, 2011. Plan provincial de développement du conseil préfectoral d'Agadir Ida Ou Tanane (2011-2016).

Raja D, Blanché C, Vallès J, 1997. Contribution to the knowledge of the pharmaceutical ethnobotany of the Segarra region (Catalonia, Iberian Peninsula). Journal of Ethnopharmacology 57: 149-160.

Rejdali M, 1996. La flore du Maroc: Etat actuel et perspectives de conservation. Actes Editions, Rabat, pp 17-22.

Saadi B, Msanda F, Boubaker H, 2013. Contributions of folk medicine knowledge in south-western Morocco: The case of rural communities of Immouzzer Ida Ou Tanane Region. International Journal of Medicinal Plant Research pp. 135145.

Salhi S, Fadli M, Zidane L, Douira A, 2010. Etudes floristique et ethnobotanique des plantes médicinales de la ville de Kénitra (Maroc), LAZAROA 31 : 133-146.

Savo V, Giulia C, Maria GP, David R, 2011. Folk phytotherapy of the Amalfi Coast (Campania, Southern Italy). Journal of Ethnopharmacology 135: 376-392.

Scherrer AM, Motti R, Weckerle CS, 2005. Traditional plant use in the areas of Monte Vesole and Ascea, Cilento National Park (Campania,
Southern Italy). Journal of Ethnopharmacology 97: 129-143

Sheldon JW, Balick M, Laird S, 1998. Is using medicinal plants compatible with conservation? Plant Talk, 13: 29-31.

Sijelmassi A, 2003. Les plantes médicinales du Maroc. 3ème édition, Edition Fenugrec, Casablanca, 285 pp.

Simbo DJ, 2010. An ethnobotanical survey of medicinal plants in Babungo, Northwest Region, Cameroon. Journal of Ethnobiology and Ethnomedicine, 6:8.

Tahraoui A, El-Hilaly J, Israili ZH, Lyoussi B, 2007. Ethnopharmacological survey of plants used in the traditional treatment of hypertension and diabetes in south-eastern Morocco (Errachidia province). Journal of Ethnopharmacology 110: 105-117.

Ugulu I, Baslar S, Yorek N, Dogan Y, 2009. The investigation and quantitative ethnobotanical evaluation of medicinal plants used around Izmir province, Turkey. Journal of Medicinal Plants Research 3: 345-367.

Yonos C, 1997. Utilisation des plantes aromatiques et des huiles essentielles en thérapeutique, Actes Editions, Rabat, pp 221-230.

Ziyyat A, Legssyer A, Mekhfi H, Dassouli A, Serhrouchni M, Benjelloun W, 1997. Phytotherapy of hypertension and diabetes in oriental Morocco. Journal of Ethnopharmacolology 58: 45-54. 